\title{
Über bleihaltigen Senf.
}

\author{
Von
}

\section{Eduard Spaeth.}

Mitteilung aus der Kgl. Untersuchungsanstalt für Nahrungs. und Genufmittel in Erlanger.

\section{[Eingegangen an 1. Oktober 1909.]}

Bei Bleivergiftungen wird oftmals, gar nicht selten aber vergeblich, nach der Ursache gefahndet, die Anlaß zu solchen Vergiftungen gegeben hat. Ich erinnere mich, daß in einer größeren Stadt solche Vergiftungen ofters aufgetreten sind, daß man die Quelle aber nicht aufzufinden vermochte. Es wurde wohl an alle möglichen Gebrauchsgegenstände gedacht, es wurden auch solche zur chemischen Untersuchung, jedoch ohne den gewünschten Erfolg herangezogen. Und doch ist, wie an dem folgenden Falle gezeigt werden soll, der Anlab zu einer solchen Vergiftung gar oft recht einfacher Natur.

Bei einer meiner Visitationen fand ich im vorigen Jabre in einem Spezereigeschäfte in einer Stadt Senftöpfe vor, die mit einem Deckel aus Metall verschlossen waren, das fast vollkommen zerstört war. Der Metalldeckel war von zahlreichen, rundlichen Löchern durchbrochen, an denen, wie an der ganzen Oberfläche des Deckels, umfangreiche Ausblühungen in Gestalt eines weißen bis weißgelben Pulvers und von zum Teil verwitterten Krystallen, zu bemerken waren. Die Senftöpfe selbst bestanden aus Porzellan oder Steingut, hatten verschiedene Formen und waren fast durchgehends mit großen Öffnungen versehen, die mit dem Metalldeckel verschlossen waren. An der Außenseite der Töpfe waren ebenfalls die Folgen der Zerstörung dieser Metalldeckel in Gestalt einer angetrockneten, weißlich gelben, verwitterten Masse zu erkennen, die anfangs abgeflossen, mit der Zeit aber eingetrocknet war. Bei der ersten Besichtigung dachte ich, daf diese Deckel wohl aus Zink besteben würden das durch die im Senf vorhandene Essigsäure zerstört worden sei; als ich aber von den reichlich vorhandenen, verwitterten. Krystallen eine geringe Menge an Ort und Stelle auf den Geschmack geprüft hatte, war mir ziemlich sicher, daß diese Metalldeckel zum größten Teil aus Blei bestehen würden.

Ich muß vorerst noch bemerken, daß diese Zerstörung der Deckel eine verschiedene war. Bei den Töpfen, die erst kürzere Zeit bezogen und im Laden aufbewahrt waren, waren diese Deckelverschlüsse nur in der Mitte mit einzelnen kleinen Löchern versehen, an denen die geschilderten Auswitterungen vorhanden waren; auf der unteren Seite waren diese Deckel meist mit einern schwarzen, feuchten Belag und ebenfalls mit Auswitterungen bedeckt. Je länger die Töpfe mit dem Senf gestanden hatten, desto intensiver war die Zerstörung der Deckel fortgeschritten, sodab es mir trotz größter Vorsicht nicht möglich war, solche Deckel, ohne sie zu zerbrechen, wegzunehmen; zudem waren die Deckel außen, wo sie die Töpfe umfaßten, infolge von gebildeten Salzen fest angeklebt. In einem Falle waren diese Metallfolien vollständig zerfressen, sodal Metallreste nur noch am Rande der Töpfe vorzufinden waren.

Nach der Entfernung der Deckel waren unter diesen in einigen Fällen ein dünnes Papier, in anderen eine dünne Pappscheibe, in einzelnen dünne Holzblätchen vorzufinden. Die Papiere waren meist vollkommen zerstört, sie waren zerbrochen und 
mit den verwitterten und auch unverwitterten Krystallen massenhaft bedeckt; die ebenfalls häufig zerbrochenen Pappscheiben und die Holzblättchen enthielten gleichfalls solche Krystalle, deren Menge sogar einige Gramm betrug. In den meisten Fällen war, ebenso wie außen am Gefäße, auch an der inneren Seite die weißliche Masse vorzufinden.

Selbst bei der vorsichtigsten Öffnung und Entfernung des Deckels, wie der Papp- und Holzscheiben, wenn diese noch gut erhalten waren, gelang es nicht, diese wegzunehmen, ohne daß der darunter befindliche Senf durch die Verwitterungsprodukte aus der bleibaltigen Folie verunreinigt wurde. In den Fällen, in denen Papierunterlagen vorhanden waren, war die Oberfläche des Senfes, wie schon eine gewöhnliche Besichtigung ergab, je nach der Länge der Aufbewahrung des Topfes mehr oder weniger stark mit den verwitterten Salzen bedeckt; in anderen Fällen war die an der inneren Seite herabfließende Bleilösung in den Senf gelangt. Solche Senfbehälter fand ich in der Folge noch in verschiedenen Geschäften vor.

Ich habe mehrere schwächer und stärker korrodierte Deckel und Büchsen mit den Deckeln entnommen und diese Deckel sowohl, wie die Verwitterungsprodukte und auch den Senf chemisch untersucht. Deckel, die sich auf einer eben erst bezogenen Sendung von Senfbüchsen vorfanden, die wenig oder gar nicht angegriffen waren, enthielten der Untersuchung zufolge 93,82, 93,40\% Blei; ein Deckel, der schon unten etwas angeätzt war, enthielt 91,17\% Blei; eine Probe eines anderen Deckels - es wurde ein Stückchen des Deckels nach Entfernung der Auswitterungen durch Abkratzen und Auswaschen mit verdünnter Säure und Wasser verwendet - enthielt nur $70,05 \%$ Blei, eine andere Probe enthielt gar nur mehr 58\% Blei.

Die reichlich vorhandenen, - in einzelnen Fällen, wie erwähnt, sogar mehrere Gramm betragenden - teils verwitterten, tells noch unverwitterten Krystalle bestanden der Untersuchung zufolge aus kohlensaurem und essigsaurem Blei. Die unter dem Deckel befindlichen Papierscheiben, die, wie gesagt, meist zerbrochen, zerfallen oder brüchig waren, waren reichlich mit den Bleisalzen getränkt; noch stärker war der Bleigehalt in den nicht selten auch noch ganz feuchten Papp- und in den Holzscheiben. Interessant war die Beobachtung, dab bei den stark verwitterten, also sebr stark zerstörten Metalldeckeln an der unteren, den Essigsäuredämpfen ausgesetzten Seite feine, düme, lamellenartige, stark metallisch glänzende Stückchen vorzufinden waren, die ich aussuchte und nach deren Behandlung mit verdünnter Säure und Wasser wieder trocknete. Durch die Untersuchung dieser Stückchen wurde festgestellt, daß diese fast aus reinem Zinn bestanden, daß bei der Zerstörung der Deckel das im Deckel reichlich vorhandene Blei gelöst und in die oben gekennzeichneten Salze übergeführt worden war, daß aber das in den Metallfoliendeekeln in ganz geringer Menge vorhandene Zinn noch größtenteils unversehrt geblieben war. Der in den Senfgefäßen vorhandene Senf war in seiner oberen Lage stets bleihaltig. In einem Falle betrug der Bleigehalt, als metallisches Blei berechnet, $0,475 \%$. Hierzu wird noch bemerkt, daß die Entfernung des Deckels und der unter dem Deckel befindlichen Papierlage sehr sorgfältig geschah und daß einzelne Stückchen der verwitterten Salze, die bei der Wegnahme des Deckels auf den oben eingetrockneten Sent gefallen waren, entfernt wurden. Nimmt man an, daß von dem Käufer eines solchen Senfes die Entfernung der Verschlüsse in Unkenntnis der Gefahr, die durch das Einfallen der gebildeten Bleisalze entsteht, unachtsam erfolgt, so liegt klar auf der Hand, daß der Bleigebalt des Senfes ein hoher, unter Umständen sogar ein sehr beträchtlicher 
werden kann und dab eine Erkrankung durch den Genuf solchen bleihaltigen Senfes sehr leicht stattfinden kann.

Die Veranlassung zur Zerstörung der bleihaltigen Metalldeckel, die nach dem Gesagten einfach aus Kapselfolien bestehen, wie sie zum Verschließen von verkorkten Weinflaschen verwendet werden, ist eine sehr naheliegende. Beim längeren Aufbewahren solcher Senfbehälter findet durch das Eintrocknen des Senfes ein Verdunsten von Wasser und von Essigsäure, ein Entweichen von Essigsäuredämpfen statt, die aus den Metallfolien unter Bildung der genannten Salze das Blei lösen. Durch die Feuchtigkeit werden die zuerst gelösten Salze in Lösung gehalten, die Salzlösung fließt nach innen und nach außen ab; mit der Zeit, wenn die Lösungen konzentrierter werden, krystallisieren diese Salze aus und verwittern allmählich an der Luft.

Ich habe nachträglich noch weiteres Untersuchungsmaterial erhalten und werde damit noch eingehendere Untersuchungen anstellen.

Daß derartige Verschlüsse aus solchem stark bleihaltigen Metall unzulässig sind, ist wohl sicher und bedarf gewiß keiner besonderen Beweisführung. Mir scheint es trotzdem nötig, einige Worte zu verlieren, da das Gesetz vom 25. Juni 1887, den Verkehr mit blei- und zinkhaltigen Gegenständen betreffend, nach mancher Richtung hin nicht ganz klar sehen läßt. Nach den technischen Erläuterungen zum Gesetzentwurfe ${ }^{1}$ ) berührt dieser nicht den Bleigehalt der Metallkapseln und Umbüllungen, die zur Verwahrung der Korke von Flaschen, Fleischextraktbüchsen und dergl. dienen, weil ein Vorgehen in dieser Richtung keineswegs geboten erscheint. Zwar hat einmal, heibt es, Wittstein ${ }^{2}$ ), nachdem er an solchen Kapseln einen hohen Bleigehalt nachgewiesen hatte, sich dahin ausgesprochen, daß diese, wenn man sie beim Öffnen der Flaschen nicht vollständig entfernt, schädlich werden können, indem sich, sobald Tropfen des Flascheninhaltes auf den Rest der bleihaltigen Umbüllung einwirken, um die Flaschenmündung herum eine Bleisolution bilde, die beim nächsten Ausschenken mit ins Trinkglas gelange und geeignet sei, Bleivergiftungen herbeizuführen. Wittstein fand in solchen Folien, die auf Flaschen, enthaltend Champagner, Likör, Senf, Mineralwasser, Bier, waren, 90,13, 91,33, 92,4, 93,1 und 94,2 bis $99,4 \%$ Blei.

Nach den technischen Erläuterungen ist die von Wittstein angegebene Befürchtung als eine übertriebene zu bezeichnen, wenigstens lassen sich keinerlei Tatsachen bezüglich des Auftretens von üblen Zufällen infolge des fraglichen Vorganges in Erfahrung bringen.

Ich möchte die von Wittstein angegebene Befürchtung keineswegs als übertrieben ansehen; mir scheint es, daß in den geschilderten Fällen viel leichter eine Bleierkrankung erfolgen kann, als durch die Benutzung eines Bierglases, dessen Deckel und Deckelfassung z. B. etwa $11 \%$ Blei enthält.

Übrigens handelt es sich in allen von Wittstein geschilderten Fällen um Gefäße, die zuerst fest mit einem Korke verschlossen waren; dieser Kork war dann mit der meist mit Pressung versehenen Folie, Metallkapsel, versehlossen. Auch ich fand Senfbüchsen ganz anderer Form, wie die in Frage stehenden, die Öffnungen im Durchmesser von vielleicht $3-4 \mathrm{~cm}$ besaßen und die mit Korken fest. verschlossen waren. Über dem Kork befanden sich Folienkapseln, die meist bleihaltig

1) R. Haas, Reichsgesetze rom 25. VI., 5. u 12. VIT. 1887. Nördlingen C. H. Beck 1887.

2) Dingler's Polytechn. Jonrnal 1873, 208, 341. 
waren; doch fand ich auch Folien mit geringem Bleigehalt vor. Hier war eine Zerstörung des Metalls und eine Verunreinigung des Senfes durch Blei möglichst ausgeschlossen, da der Topf fest mit einem ziemlich starken Kork verschlossen war. Ganz anders liegen aber die Dinge bei den von mir gefundenen Gefäßen, die eine Öffnung im Durchmesser von $8-10 \mathrm{~cm}$ hatten und die, wie mitgeteilt, mit der Metallfolie und mit nur einem darunter befindlichen Papier, Papierkarton oder Holzblättchen versehlossen waren, die sämtlich, wie die Praxis ergeben hat, weder vor einer Zerstörung der stark bleihaltigen Folie, noch vor einer Verunreinigung und Vergiftung des Inhaltes der Gefäße mit den Bleisalzen schützen.

Der Senf ist als eine Konserve zu betrachten, die gerade infolge ihrer Zusammensetzung bei längerer Lagerung auf bleihaltige Metalle oder bleihaltige Geschirre unter allen Umständen zerstörend und bleilösend einwirkt. Auf die bleihaltigen Verschluß. deckel wirkt, wie wir gesehen haben, die im Senf vorhandene Essigsäure beim Verdunsten und Entweichen in viel stärkerem Maße ein, als dies durch die Einwirkung des Senfes direkt auf ein bleihaltiges Lot der Fall sein dürfte; würde der Senf in einem verzinnten Gefäße, in einer Konservenbüchse verwahrt, so würde von der Verzinnung eben die gesetzlich vorgeschriebene Beschaffenheit verlangt werden; dies gilt sowohl von der Büchse, wie von dem dazu gehörigen Deckel. Das gleiche muß von dem VerschluBdeckel verlangt werden, wenn or in der angegebenen Weise angebracht ist, er darf einen Bleigehalt von $1 \%$ nicht übersteigen, abgesehen davon, daß derartige Verschlüsse sich in diesem Falle überhaupt nicht eignen.

Die unter der Bleifolie befindlichen Unterlagen sind ohne jeden Schutz und verhindern die Zerstörung der Folien und die Vergiftung des Senfes nicht im geringsten.

Wir wissen, - was in den mehrfach erwähnten technischen Erläuterungen ausgegeführt wird -- daß man wiederholt ohne Erfolg versucht hat, das Blei entweder durch Zwischenlagen von Papier oder durch Plattieren der Bleifolie mit reinem Zinn unschädlich zu machen. Nach Untersuchungen von Feichtinger ${ }^{1}$ ) und Pettenk ofer schützen z. B. weder Zwischenlagen von Papier, noch Verzinnungen von Bleihüllen vor dem Angriffe und vor der Lösung des Bleies aus den Folien, z. B. durch Schnupftabak. Aus allen diesen Gründen muß doch wohl geschlossen werden, daß von derartigen Metallfolien, wenn sie als Verschlüsse für besagte Zwecke benutzt werden, verlangt werden $\mathrm{muß}$, daß sie nicht mehr wie $1 \%$ Blei enthalten.

Man könnte nun vielleicht einwenden, daß der Gesetzgeber ja eigentlich alle Gegenstände aufgeführt hat, die den gesetzlichen Vorschriften zu genügen haben. Er erwähnt die EB-, Trink- und Kochgeschirre und Flüssigkeitsmaße, die Druckvorrichtungen zum Ausschank von Bier u. s. W., die Metallteile für Kinder-Saugflaschen, die Geschirre und GefäBe zur Verfertigung von Getränken und Fruchtsäften, die Konservenbüchsen, Gefäße, in denen sich Rückstände von bleihaltigem Schrote befinden, die zur Verpackung von Schnupf- und Kautabak sowie von Käse dienenden Metallfolien. Nicht erwähnt werden allerdings solche aus fast reinem Blei bestehenden Deckel für Senfbüchsen; es ist auch im Gesetze nicht gesagt, wie die zur Verwahrung von Nahrungsmitteln dienenden Gefäße beschaffen sein müssen; es ist nur die Rede von Geschirren, die zur V erferti gun g von Getränken und Fruchtsäften verwendet werden.

Der Grundzug dieses Gesetzes, wie des Nahrungsmittelgesetzes ist aber doch

1) Dingler's Polytechn. Journal 103, 149.

) Ärztl. Intelligenzblatt 1859, 127. 
zweifellos der, das Publikum vor Gefahren, die ihm durch den Genuß von Nahrungsmitteln, die infolge ihrer Zubereitung oder Aufbewahrung in bleihaltigen Gefäßen bleihaltig werden, drohen, zu schützen. Zu einem Aufbewahrungsgefäße gehört ohne Zweifel auch der Verschlub, gerade wie das Gesetz anerkannt hat, daß nicht nur die Metallderkel der Biergläser und Steingutkrüge, sondern auch deren Scharniere, Gewinde, Befestigungsringe an den Henkeln voll und ganz dem Gesetze zu genügen haben.

Allerdings möchte ich an die seinerzeit im Handel befindlichen stark bleihaltigen Faßhähne erinnern, die zum Abfüllen von Wein, Essig u. s. w, angeboten wurden und die einen Bleigehalt bis 60 und $70 \%$ aufwiesen. Man war sich ${ }^{1}$ ) nicht recht klar, ob das Nahrungsmittelgesetz oder das Blei-Zink-Gesetz auch diese Gegenstände, die ja nicht ausdrücklich im letzteren benannt sind, auszudehnen wäre. Nach einem Urteil des Kgl. Oberlandesgerichtes München ${ }^{2}$ ) vom 17. Juni 1893 geht die Absicht des Gesetzes nicht dahin, den gesamten Handel und Verkehr mit blei- und zinkbaltigen Gegenständen zu regeln. Das Gesetz, sagt dieses Gericht, hat nur einzelne Gegenstände herausgegriffen, bei deren Herstellung, Verpackung u. s. w. das Blei nicht oder nur in bestimmt begrenztem Gewichtsverhältnisse zur Verwendung kommen darf. Für die übrigen Gegenstände, bei deren Anfertigung oder Aufbewahrung eine nachteilige Einwirkung auf die Gesundheit durch Anwendung von Blei nicht weniger zu befürchten ist, sollte die vorsorgende Einwirkung der Landesgesetzgebung keineswegs beseitigt werden.

Bis zu einer Revision des Gesetzes, die nach dem Gesagten recht notwendig erscheint, müßte den Gefahren durch entsprechende andere Maßnahmen, z. B. durch oberpolizeiliche Vorschriften, entgegengetreten werden. Wohl alle bayerischen Kreisregierungen haben deswegen schon in den oberpolizeilichen Vorschriften die Verwendung von Metallhähnen bei der Verzapfung von Wein, Branntwein und Essig überhaupt verboten. In die gleichen Vorschriften für Uuterfranken und Aschaffenburg ist. ein Paragraph aufgenommen, der auch in dem von mir besprochenen Falle Wandel schaffen würde; es heißt: „Überhaupt müssen Geschirre, Gefäße, Geräte, Umhüllungen aller Art, die bei ihrem Gebrauche in irgend einer Weise mit Nahrungs- und Genubmitteln in fester oder flüssiger Form in Berührung kommen, in bezug auf das Material, aus dem sie bestehen (soweit dies zink- oder bleihaltig ist), ferner in bezug auf Verzinnung, Lötung nnd Glasierung oder Emailliernng den Vorschriften des Reichsgesetzes vom 25. Juni 1887 entsprechen."

Es wäre gewiß sehr zu wünschen, wenn bei einer Revision des Gesetzes in klarer Weise das Verbot der Verwendung von bleihaltigem Metall und von bleiabgebenden Geschirren zur Verwahrung von Nahrungsmitteln, zumal wenn diese infolge ihrer Zusammensetzung lösend und zerstörend auf das Blei einwirken, ausgesprochen würde. Der besprochene Fall mag zeigen, wie leicht das konsumierende Publikum den Gefahren einer Erkrankung durch Aufnahme von Blei und Bleisalzen ausgesetzt. sein kann. Um sich einen Vorteil zu verschaffen, da solche fast nur aus Blei be. stehende Deckel wesentlich billiger sind als solche aus Zinn, wird die Gesundheit. der Abnehmer in unverantwortlicher Weise aufs Spiel gesetzt; der Hersteller solcher Senfgefäße muß wissen, daß sich solche Verschlüsse, die in der hier geschilderten Weise angebracht sind, nicht eignen.

1) A. Würzburg, Die Nahrungsmittelgesetzgebung. Leipzig 1894.

2) Daselbst S. 276; Sammlung von Entscheidungen 7, 404. 
Meiner Ansicht nach muB nicht immer erst klinisch ein Fall bekannt sein, in dem die Bleierkrankung mit aller Sicherheit z. B. auf den Genuß eines in der angegebenen Weise aufbewahrten Senfes zurückgeführt werden kann; ich wiederhole, was. ich im Eingange dieser kurzen Mitteilung gesagt habe: In vielen Fällen von Bleierkrankungen wird man, wie die Erfahrung seither gezeigt hat, der Ursache der Vergiftung nicht mehr auf die Spur kommen können.

\title{
Ergebnisse der Untersuchung von Heilmitteln, Geheimmitteln, kosmetischen und ähnlichen Mitteln.
}

\section{Von}

\author{
A. Juckenack und C. Griebel.
}

Mitteilung aus der Statichen Anstalt zur Untersuchung von Nahrangsund Genufmitteln sowie Gebrauchsgegenständen für den Landespolizei. bezirk Berlin.

\section{[Eingegangen am 17. Oktober 1909.]}

Im AnschluB an unsere einschlägigen früheren Mitteilungen ${ }^{1}$ ) bringen wir einen. weiteren kurzen Auszug aus den Ergebnissen unserer Untersuchungen auf diesem Gebiete, indem wir zugleich auf die früher gemachten allgemeinen Ausführungen. Bezug nehmen.

176. Cascarino, Entfettungstee von Apotheker Hugo Storz in. Berlin, bestand aus einem Gemenge zerkleinerter Vegetabilien. Festgestellt wurden: Folia Sennae, Folliculi Sennae, Flores Sambuci, Rhizoma Rhei, Radix Valerianae, Fucus vesiculosus, Cortex Frangulae, Cortex Cascarae sagradae, Radix Taraxaci cum. herba, Fruetus Anisi und Fruetus Foenieuli. - Ein Karton mit etwa $140 \mathrm{~g}$ Inhalt. kostete $1,75 \mathrm{Mk}$.

177. Hustentropfen Frebar, früher Regina, sollten ein Destillat aus. Benzoe, Campher, Alant, Salmiak, kohlensaurem Kali, Anis, Wasser und Spiritus. sein. Die aromatische, flüchtige, ammoniakfreie alkoholbaltige Flüssigkeit ließ im. Geruch vorwiegend Nelkenöl erkennen. - $30 \mathrm{~g}$ kosteten $0,50 \mathrm{Mk}$.

178. Nerventropfen Frebar, früher Regina, waren der Angabe des Fabrikanten entsprechend ein alkoholhaltiges Destillat aus Baldrian und Campher. $50 \mathrm{~g}$ kosieten $1,00 \mathrm{Mk}$.

179. Scheuertee, Mittel gegen Diabetes von S. Scheuer Nachfolger A. Camphausen in Berlin-Wilmersdorf, bestand aus mittelfein gepulverten Boldoblättern, die zu Tabletten komprimiert waren. - Preis eines Kartons. mit 250 Tabletten zu je $1 \mathrm{~g}=12,50 \mathrm{Mk}$.

180. Arekanub-Bandwurmmittel von $O$. Wichert in Berlin bestand aus einer Glasröhre mit $6 \mathrm{~g}$ Arekanußpulver sowie aus 6 Gelatinekapseln mit Ricinusöl von je 2,75 g Gewicht. - Prejs 2,50 Mk.

1) $\mathrm{Vgl}$, diese Zeitschrift 1907, 14, 498-499 und 1909, 17, 79-86. 